\title{
On the Regional Stabilization of Locally Unstable Predator-Prey Relationships
}

\author{
JOHN H. VANDERMEER \\ Department of Zoology, \\ University of Michigan, \\ Ann Arbour, Michigan 48104, U.S.A.
}

(Received 9 November 1972)

\begin{abstract}
The stabilizing effects of interhabitat migration and local population extinction are established by an analysis of the variable $P_{t}$, where $P_{t}$ refers to the proportion of habitats occupied by species $i$. This is followed by an analysis of the qualitative nature of changes in regional and local factors and their influence on establishing stable or unstable conditions. It is seen that the appropriate balance between interhabitat migration and local population extinction is capable of stabilizing an otherwise unstable predatorprey interaction in a wide variety of situations.
\end{abstract}

\section{Introduction}

The original equations of Lotka (1925) and Volterra (1926) relate predator density to prey density and predict structurally stable permanent oscillations. These solutions are unrealistic from a biological point of view because the trajectories are dictated by the boundary conditions. Addition of density dependent negative feedback to each population yields a system which exhibits damped oscillations. However, at least a few real cases are known where populations of predator and prey undergo oscillations of ever increasing amplitude until the system goes extinct. In fact it is this qualitative result which is most commonly obtained in artificially constructed laboratory situations of predator-prey interactions. Rozenzweig \& MacArthur (1963) extended these basic equations by exploring the qualitative effects of adding positive density dependent feedback to the prey population. This results in unstable oscillatory conditions, as commonly observed in most laboratory systems. Thus, in general, mathematical models give the qualitative results that predator-prey systems should have damped oscillations if both predator and prey exhibit intraspecific negative density dependent feedback, unstable oscillations if the prey exhibits positive density dependent feedback.

Lloyd (personal communication) has shown, for arthropod communities т.в. 
occupying beach litter in England, that the process of local extinction is important in maintaining species diversity. This suggests that unstable biological conditions may exist for local geographical areas, yet extinctions over a larger region are not evident due to migration from neighboring areas. Two recent papers (Levins \& Culver, 1971; Horn \& MacArthur, 1972) have provided possible mechanisms for the stabilization of potentially unstable competitive interactions. Following the basic ideas introduced in those two papers, the present communication shows how an unstable predator-prey oscillation can be stabilized under appropriate environmental heterogeneity. Further comments will then be made on the effect of variable utilizations of the environment by the predator and the prey, and the effect that this might have on the stabilization of the inherently unstable system.

\section{Qualitative Results}

The model follows the procedure of Levins \& Culver (1971) and Horn \& MacArthur (1972) in considering an environment composed of discrete habitat units some of which are occupied by the predator, some by the prey, some by both, and some by neither. Let $p$ be the proportion of the habitats that are occupied by the predator and $q$ the proportion that are occupied by the prey; what are the circumstances which lead to $p$ and $q$ having positive stable values after the system reaches equilibrium? The conditions leading to stability are related to migration and extinction rates for both predator and prey, and the way in which migration rates are related to total habitat space.

Given an environment of a particular size (i.e. a particular number of habitats), suppose that there exists an unstable predator-prey pair in each of the habitats. Suppose further that each of the species migrates from habitat to habitat at a given rate, and goes extinct locally at a particular rate. Let the extinction of prey populations be proportional to the proportion of habitats occupied by predators and the extinction of predator populations be proportional to the proportion of habitats not occupied by prey populations. Qualitatively such a system can be stable in the sense that a constant proportion of habitats will be occupied by both predator and prey, even though each individual habitat contains an unstable system. The qualitative generalizations are that the system will tend to become regionally stabilized (i) as the predator's migration rate decreases, (ii) as the predator's extinction rate increases, (iii) as the prey's migration rate increases, and (iv) as the prey's extinction rate decreases.

A further set of qualitative conclusions has to do with changing migration and extinction rates as functions of generalized environmental factors. We can imagine two sorts of factors which might operate-regional factors and 
local factors. Since extinction rates have to do with the dynamics of the predator-prey interaction as it exists within individual habitats, we conceive of local factors as affecting extinction rates. Similarly we conceive of regional factors as affecting migration rates.

First consider factors which might operate at a regional level, such as an increase in the effective distance between habitats or barriers introduced between habitats. Beginning with an initially unstable system, the regional factor might operate either to stabilize an unstable system or destabilize a stable system. Stabilization of an unstable system will occur if the predator's migration rate decreases more rapidly than does that of the prey, or if the prey's rate of migration increases more rapidly than does that of the predator. As the prey's migration rate becomes large relative to that of the predator, the system tends to be stabilized.

Next consider the effect of changing local conditions. The way in which organisms move among the habitats remains constant, but within each habitat something happens to change the local extinction rates. Again beginning with an unstable system, increase in the predator extinction rate or decrease in the prey extinction rate tends to stabilize the system.

\section{Details of the Model}

Consider the following system of equations:

$$
\begin{aligned}
& \frac{\mathrm{d} p}{\mathrm{~d} t}=\lambda_{1} p(1-p)-\mu_{1}(1-q) \mathrm{p}, \\
& \frac{\mathrm{d} q}{\mathrm{~d} t}=\lambda_{2} q(1-q)-\mu_{2} p q,
\end{aligned}
$$

where $p$ refers to the proportion of habitats occupied by the predator, $q$ refers to the proportion of habitats occupied by the prey (note that $p+q$ is not necessarily equal to one), $\lambda_{1}$ is equal to the migration rate of the predator, $\lambda_{2}$ is equal to the migration rate of the prey, $\mu_{1}$ is equal to the extinction rate of the predator and $\mu_{2}$ is equal to the extinction rate of the prey. The first term in both of the above equations simply stipulates the way in which the environment becomes saturated, given a constant interhabitat migrating propensity. The second term in the first equation gives the proportion of those habitats occupied by predators that are likely to be rendered predatorless, $\mu_{1}(1-q)$. The second term in the second equation gives the proportion of those habitats occupied by the prey which are likely to be rendered preyless, $\mu_{2} p$. Setting the derivatives equal to zero and cancelling constant multipliers we obtain 


$$
\begin{aligned}
& p=\frac{\lambda_{1}-\mu_{1}}{\lambda_{1}}+\frac{\mu_{1}}{\lambda_{1}} q\left(\frac{\mathrm{d} p}{\mathrm{~d} t}=0\right), \\
& p=\frac{\lambda_{2}}{\mu_{2}}-\frac{\lambda_{2}}{\mu_{2}} q\left(\frac{\mathrm{d} q}{\mathrm{~d} t}=0\right) .
\end{aligned}
$$

Note in the above equations that when $p=0, q=1$ for equation (2) and when $q=1, p=1$ for equation (1). Given these constraints on the system it can be seen that there are two possible outcomes, both illustrated in Fig. 1. In case 1 we have a system which oscillates, the oscillations damping ultimately
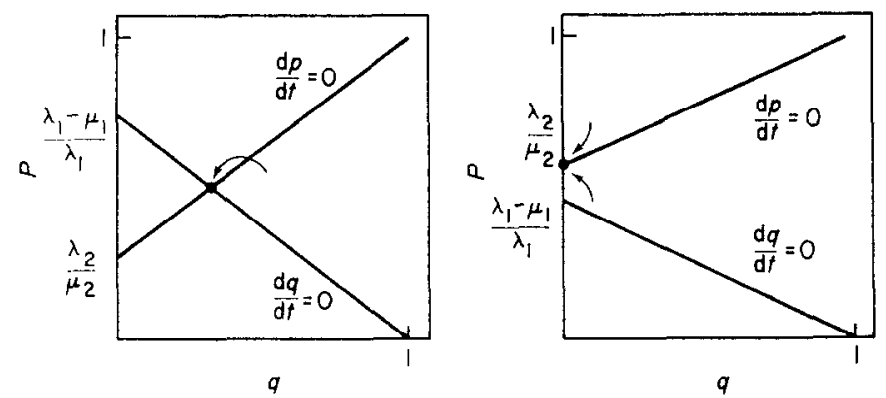

Fig. 1. The two possible solutions for the basic equations.

to an equilibrium point. In case 2 we have an unstable system where the system degenerates to zero prey populations. The condition under which case 1 (stability) is obtained is given as

$$
1<\frac{\lambda_{2}}{\mu_{2}}+\frac{\mu_{1}}{\lambda_{1}},
$$

while reversal of the inequality stipulates the condition for case 2 . The qualitative results as described in the previous section are easily derived from relation 3 or Fig. 1. Relation 3 is graphed in Figs 2 and 3 (note that whenever $\lambda_{2}>\mu_{2}$ the system is stable). The shaded area in Fig. 2 represents those combinations of $\lambda_{1}$ and $\lambda_{2}$ for which the system as a whole will be stable and the shaded area in Fig. 3 represents those combinations of $\mu_{1}$ and $\mu_{2}$ for which that system will be stable.

We now consider the effects of regional changes in the system. That is, suppose the $\mu$ 's remain constant and the $\lambda$ 's change as a function of some regional factor. In Fig. 4 are pictured all possible cases. If both predator and prey have their migration rates decreased by the same factor, we go from point $A$ toward point $B$ in Fig. 4, and the system will tend to destabilize. Note that this is usually the case even if both $\lambda_{1}$ and $\lambda_{2}$ decrease at exactly the same rate, a consequence of the fact that the function which separates 


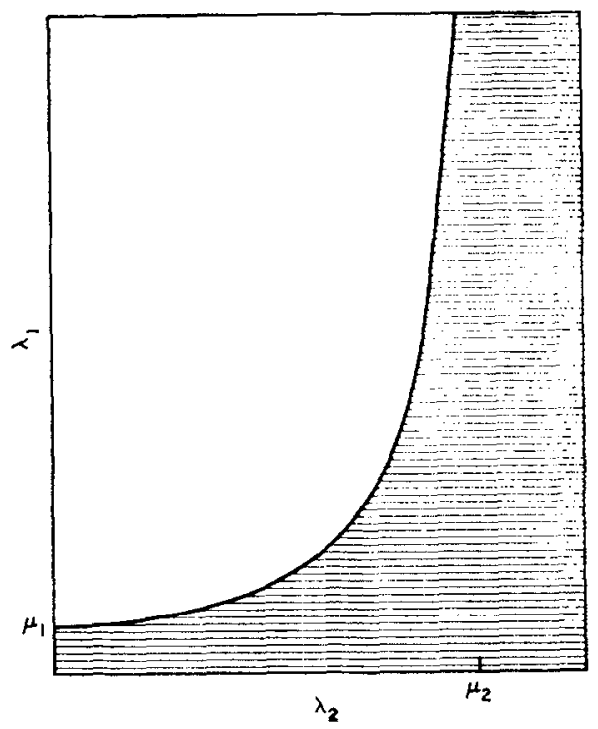

FIG. 2. Graph of relation (3) in $\lambda_{1}, \lambda_{2}$ space.

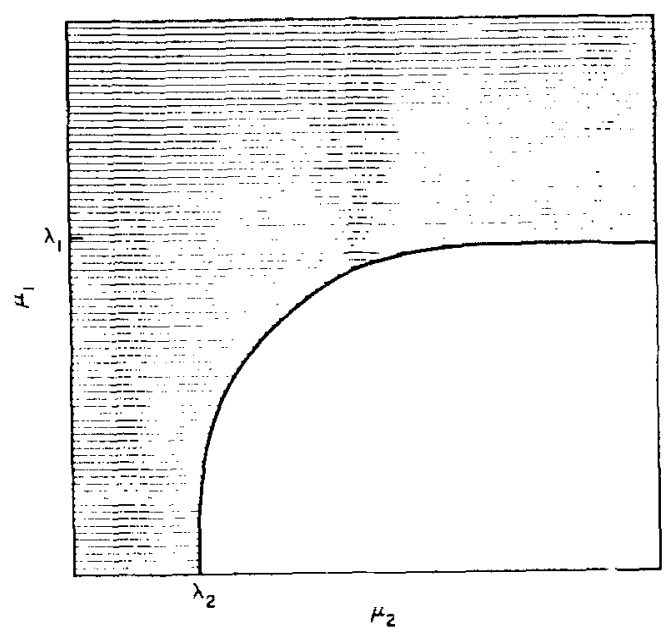

Fig. 3. Graph of relation (3) in $\mu_{1}, \mu_{2}$ space.

the stable from the unstable space is concave. If the predator's rate decreases much faster than the prey's we go from point $B$ to point $C$, from an unstable system to a stable system. On the other hand, beginning with the unstable system at point $\mathbf{B}$, if the predator's rate decreases less rapidly than the prey's, 


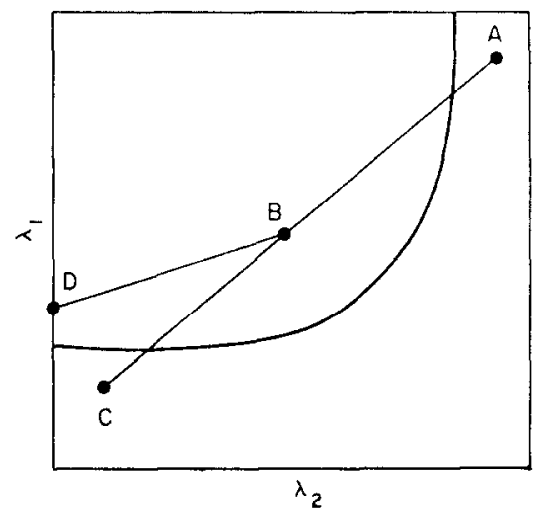

FIG. 4. Changes from stable to unstable as a function of regional factors.

we go from point $B$ to point $C$ and the system will never become stabilized. Thus, particular adjustments of regional factors can either destabilize a stable system or stabilize an unstable system. It should be recalled that stable or unstable in this context refers to regional stability, all systems in this model being locally unstable.

It is interesting to pursue the special case in which both migration coefficients decrease at the same rate as a function of the regional factor. This is equivalent to supposing that the migration rates correspond to the equation, $\lambda_{1}=a+\lambda_{2}$, where $a$ is constant referring to the value that $\lambda_{1}$ will have when $\lambda_{2}$ is equal to 0 . The constant $a$ can be positive or negative. We thus seek those values of $a$ for which all combinations of $\lambda_{1}$ and $\lambda_{2}$ which satisfy this linear relationship fall within the area of stability. Substituting the linear relationship, $\lambda_{1}=a+\lambda_{2}$, into equation (3) we obtain

$$
\lambda_{2}=\frac{1}{2}\left(a-\mu_{2}\right) \pm \frac{1}{2} \sqrt{a^{2}+2 \mu_{2} a+\mu_{2}^{2}}-4\left(\mu_{1} \mu_{2}\right) .
$$

The value of $\lambda_{2}$ which gives equal roots to the above equation stipulates the limiting value of $a$ which separates the linear relationships which are always stable from those which are sometimes stable and sometimes unstable. Applying the quadratic equation we see that this value is given by,

$$
a=-\mu_{2} \pm 2 \sqrt{\mu_{1} \mu_{2}} \text {. }
$$

The larger of these two values corresponds to the root of $\lambda_{2}$ which obtains when $\lambda_{1}$ is greater than 0 . Thus we see that if the predator's migration rate has a value less than $2 \sqrt{\mu_{1} \mu_{2}}-\mu_{2}$ when the prey's migration rate is zero, the system cannot be stabilized by a regional factor which affects both predator and prey migration rates in the same way. If the value of the predator migration rate when the prey's migration rate is zero is greater than that 
value but less than $\mu_{1}$, an unstable system can be stabilized, either by increasing or decreasing migration rate. Furthermore by consistent changes in the migration coefficients, a stable system can be pushed first into an unstable system and further pushed into a stable system. These relationships are presented in Fig. 5.

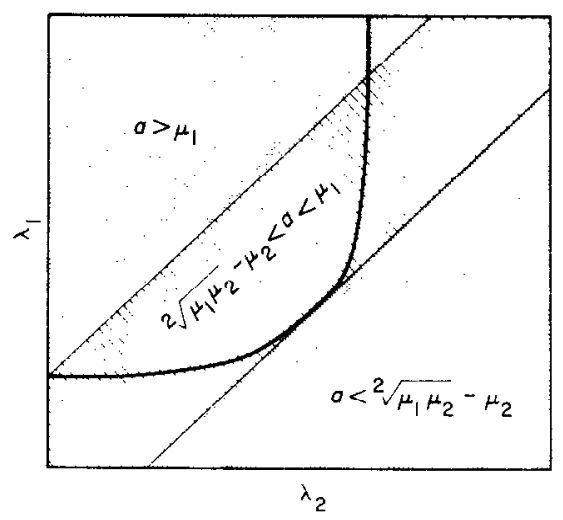

FIG. 5. Regions of $\lambda_{1}, \lambda_{2}$ space in which a regional factor can or cannot destabilize the system (see text for explanation).

Considering now the changes which might be effected by the application of a local factor changing in the system, we see that the results are perfectly analogous with the results of changing the regional factor. In Fig. 6 those results are presented. As we go from point A to point $B$ we go from a stable to an unstable system, from point $B$ to $C$ from an unstable to a stable system and from B to D from an unstable system to an unstable system. Any local factor which operates in such a way that the extinction coefficients go from point $B$ to point $D$ in Fig. 3 is a factor which cannot stabilize the system.

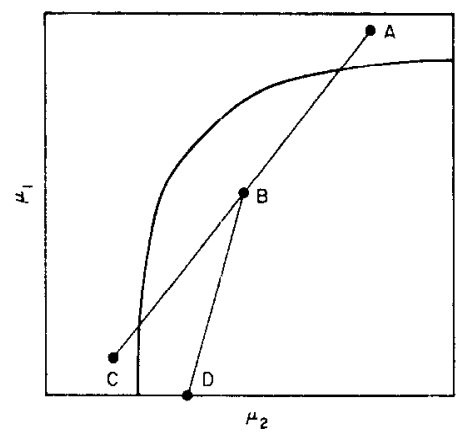

Fig. 6. Changes from stable to unstable as a function of local factors. 
Similar to the previous analysis we can investigate the consequences of a local factor which operates to change the extinction rate for the predator in the same way that the extinction rate for the prey is changed. The results are analogous to the same analysis for the migration coefficients, and are presented graphically in Fig. 7.

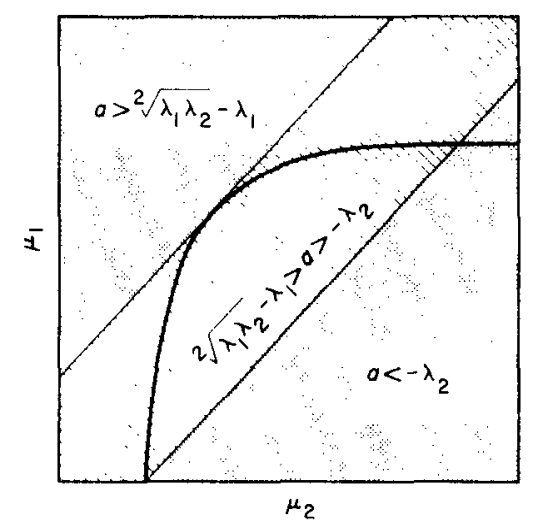

FIG. 7. Regions of $\mu_{1}, \mu_{2}$ space in which a local factor can or cannot destabilize the system (see text for explanation).

\section{Hidden Assumptions of the Model}

The model presented in this paper as well as the similar model presented by Levins \& Culver (1971) and Horn \& MacArthur (1972) makes certain tacit assumptions about the manner in which migrations occur. In order to see these assumptions we consider the following simple probability model. Let $p_{i}(t)$ equal the probability that the $i$ th habitat will be occupied by the predator at time $t$, and $q_{i}(t)$ be the probability that the $i$ th habitat will be occupied by the prey at time $t$. Then we can write the following recursion equations to describe the process of predation in a system of $H$ habitats

$$
\begin{aligned}
p_{i}(t)=p_{i}(t-\Delta t) q_{i}(t-\Delta t)+\left[1-p_{i}(t-\Delta t)\right] & \\
\times & \times \sum_{j=1}^{H} g_{i j} p_{j}(t-\Delta t)-\mu_{1}^{\prime} p_{i}(t-\Delta t)\left[1-q_{i}(t-\Delta t)\right], \\
q_{i}(t)=q_{i}(t-\Delta t)\left[1-p_{i}(t-\Delta t)\right]+ & {\left[1-q_{i}(t-\Delta t)\right] } \\
& \times \sum_{j=i}^{H} g_{i j}^{\prime} q_{j}(t-\Delta t)-\mu_{2}^{\prime} q_{i}(t-\Delta t) p_{i}(t-\Delta t),
\end{aligned}
$$

where $\mu^{\prime}$ is analogous to $\mu$ in the previous equation (the exact relationship will be shown momentarily) and $g_{i j}$ and $g_{i j}^{\prime}$ refer to the probability of migrating from habitat $j$ to habitat $i$. If we presume that $p_{i}=p_{j}$, that is, the pro- 
bability of a habitat being occupied by a predator is the same over all habitats, and likewise, $q_{i}=q_{j}$, and subtracting $p_{i}(t-\Delta t)$ from both sides of the first equation and $q_{i}(t-\Delta t)$ from both sides of the second equation,

$$
\begin{aligned}
& p_{i}(t)-p_{i}(t-\Delta t)= \\
& \quad\left(\sum g\right) p_{i}(t-\Delta t)\left[1-p_{i}(t-\Delta t)\right]-\left(1+\mu_{1}^{\prime}\right) p_{i}(t-\Delta t)[1-q(t-\Delta t)], \\
& q_{i}(t)-q_{i}(t-\Delta t)= \\
& \sum g^{\prime} q_{i}(t-\Delta t)[1-q(t-\Delta t)]-\left(1+\mu_{2}^{\prime}\right) p_{i}(t-\Delta t) q_{i}(t-\Delta t),
\end{aligned}
$$

and letting $\Delta t$ become very small we can write,

$$
\begin{aligned}
& \frac{\mathrm{d} p}{\mathrm{~d} t}=\lambda_{1} p(1-p)-\mu_{1} p(1-q), \\
& \frac{\mathrm{d} q}{\mathrm{~d} t}=\lambda_{2} q(1-q)-\mu_{2} q p,
\end{aligned}
$$

where $\lambda_{1}=\sum g, \lambda_{2}=\sum g^{\prime}, \mu_{1}=1+\mu_{1}^{\prime}$, and $\mu_{2}=1+\mu_{2}^{\prime}$. Thus, we obtain the original set of differential equations. However, by making the assumption that $p_{i}=p_{j}$ and $q_{i}=q_{j}$, certain assumptions about the way in which the $g_{i j}$ behave have been made. We must either presume that the $g_{i j}$ are equal over all $j$, or that the values of the $g$ 's are randomly allocated amongst habitat pairs at each $\Delta$ time unit. That is, in order for us to presume that the probability of finding individuals in any one habitat is the same over all habitats we must also presume that the probability of migrating from any one habitat to any other habitat is equal, or that the probabilities of migrating from habitat to habitat are drawn at random from some population, the mean of which is equal to the $\lambda$ of the original differential equations.

\section{Discussion}

A model has been presented for the exploration of conditions which provide for the stabilization of locally unstable predator-prey situations. Beginning with a series of local predator-prey associations, each of which is unstable, equations are derived involving rates of migration from habitat to habitat and local extinction rates. Summarizing the qualitative results of the model, as the predator rate of migration becomes small in comparison with the rate of migration of the prey, the system tends to be regionally stable, and as the extinction rate of the predator becomes large relative to the extinction rate of the prey, the system becomes stable.

The consequences of introducing potential stabilizing factors into the system are variable. For example, a potentially stabilizing factor which acts in such a way that the extinction rate of the predator is increased faster than that of the prey, results in the stabilization of a basically unstable system. This is exactly what was observed by Gause (1934) in the system where a flocculent 
material was added to the bottoms of test tubes which contained a Paramecium-Didinium unstable system. The flocculent material provided refugia for the Paramecium, thus decreasing its population extinction rate. As predicted by the model, that system tended to be stable. To the extent that the extinction rates tend toward zero we may be concerned with a locally stable situation and the consequences as presented in this paper are not particularly applicable. As hiding places are introduced for the prey such that predation intensity is less at low prey densities than it is at high densities, the extinction rate of the prey will go down in any local habitat. This will result in the stabilization of the system.

Considering the effect of a potential stabilizing factor on the migration rates, we note that if the migration rate of the predator decreases faster than that of the prey, the system becomes stabilized. For example, the construction of predator barriers between habitats, such that the predators have their migration rates decreased, is likely to stabilize the system. This is precisely the consequence that was observed in the experiments of Huffaker (1958) in which the predacious mite Typhlodromus occidentalis was not able to cross vaseline trails as easily as the herbivorous prey species, Eotetranychus sexmaculatus. This system seemed to be more or less stabilized, whereas it had been shown previously that the system was unstable in any local area.

The model presented in this paper has the rather stringent assumption that migration rates are either randomly dispersed over habitat pairs or constant over habitat pairs. Exactly what effect a different pattern of migration will have on the qualitative consequences presented herein is not known. Currently investigations are underway to simulate such a system. It is thought that even though the basic differential equations are not truly applicable, various different patterns of migration can be accounted for by varying the rates of migration.

I wish to express my sincere gratitude to the students in Zoology 542 at the University of Michigan, whose conversations led to the ideas developed herein, and to the ecology group at the University of Michigan who permitted my use of their profound criticisms. Bob Gleasner, Steve Hubbell, Nelson Hairston and Dave Titman are specifically to be thanked for their contribution to a number of the ideas presented herein.

\section{REFERENCES}

GaUSE, G. F. (1934). The Struggle for Existence. Baltimore: Williams and Wilkins.

HORN, H. \& MACARTHUR, R. H. (1972). Ecology 53, 749.

HUFFAKER, C. B. (1958). Hilgardia 27, 343.

Levins, R. \& Culver, D. (1971). Proc. natn. Acad. Sci. U.S.A. 68, 1246.

LOTKA, A. J. (1925). Elements of Physical Biology. Baltimore: Williams and Wilkins.

Rosenzweig, M. L. \& MACArthur, R. H. (1963). Am. Nat. 97, 209.

Volterra, V. (1926). Memorie Accad. pont. Nuovi Lincei, 2, 31. 